\title{
Effects of the circ_101238/miR-138-5p/CDK6 axis on proliferation and apoptosis keloid fibroblasts
}

\author{
DAN YANG ${ }^{1}$, MINGJIN LI ${ }^{2}$ and NA DU ${ }^{3}$ \\ ${ }^{1}$ Department of Dermatology, The First Affiliated Hospital of Jinzhou Medical University, Jinzhou, Liaoning 121001; \\ ${ }^{2}$ Department of Dermatology, Liaoning Jinqiu Hospital, Shenyang, Liaoning 110015; ${ }^{3}$ Department of Cardiology, \\ The First Affiliated Hospital of Jinzhou Medical University, Jinzhou, Liaoning 121001, P.R. China
}

Received November 28, 2019; Accepted April 29, 2020

DOI: $10.3892 /$ etm.2020.8917

\begin{abstract}
The formation of keloid scars is normally induced by cutaneous injuries, however, the detailed mechanisms underlying keloid formation remain largely unknown. The present study aimed to investigate the effects of circular RNA_101238 (circ_101238) on the proliferation and apoptosis of keloid fibroblasts and to identify the underlying molecular mechanisms of these effects. Reverse transcription-quantitative (RT-q)PCR was performed to determine the expression levels of circ_101238, microRNA (miRNA/miR)-138-5p and cyclin-dependent kinase 6 (CDK6) in keloids and normal skin tissues. Following transfection with short hairpin (sh)-circ_101238, LV-circ_101238, miR-138-5p mimics, miR-138-5p inhibitors and small interfering (si)-CDK6, cell proliferation was assessed using a cell counting kit- 8 assay. Furthermore, cell apoptosis was evaluated via flow cytometric analysis, while a dual-luciferase assay was performed to confirm interactions between circ_101238, miR-138-5p and CDK6. The expression levels of the proliferation marker, CDK6 and apoptosis marker, caspase-3 were determined via RT-qPCR and western blot analyses. The results demonstrated that expression levels of circ_101238 and CDK6 were significantly increased in keloid samples, while miR-138-5p expression was reduced in comparison to normal skin. Furthermore, circ_101238 was demonstrated to bind miR-138-5p, which subsequently targeted CDK6. Proliferative activity and CDK6 expression were significantly decreased in keloid fibroblasts following transfection with sh-circ_101238 or miR-138-5p mimics, while cell apoptosis was markedly increased. Furthermore, co-transfection with miR-138-5p mimics reversed the effects caused by overexpression of circ_101238. Treatment of keloid fibroblasts
\end{abstract}

Correspondence to: Dr Na Du, Department of Cardiology, The First Affiliated Hospital of Jinzhou Medical University, 2 Renmin Road, Jinzhou, Liaoning 121001, P.R. China

E-mail: dunaduan87@gmail.com

Key words: circular RNA_101238, micoRNA-138-5p, cyclindependent kinase 6 , keloid, fibroblast, proliferation, apoptosis with si-CDK6 counteracted the biological behavior changes induced by miR-138-5p inhibitors. Additionally, transfection with LV-CDK6 reversed the effects caused by miR-138-5p mimics. Taken together, the results of the present study demonstrated that circ_101238 was upregulated in keloid tissues in comparison with normal tissues and that circ_101238 knockdown inhibited cell proliferation, while promoting apoptosis of keloid fibroblasts via the miR-138-5p/CDK6 axis. These results suggest that circ_101238 may serve as a promising therapeutic candidate for keloid therapy and that circ_101238/miR-138-5p/CDK6 signaling has the potential to regulate the growth of keloid fibroblasts.

\section{Introduction}

Keloids are a type of abnormal scars caused by cutaneous injuries, such as trauma, burns and surgery $(1,2)$. Increased levels of collagen I and III are produced by fibroblasts from the growing margin of keloids, compared with intra-/extra-lesional areas (1). Keloids, which overgrow the boundaries of the original injuries, are characterized by itchiness and pain (2). A previous study reported that several gene regulators, including cytokines and chemokines are involved in the initiation and progression of keloid development (3). However, the molecular mechanisms underlying initiation and development of keloids remain unclear, thus therapeutic strategies are yet to be developed for patients with keloids.

Circular RNAs (circRNAs) are non-coding RNAs, which form a continuous circle and are more stable than linear RNAs (4,5). Although some circRNAs, such as CDR1as, are considered to be putative gene regulators (4), the detailed functions of the majority of circRNAs remain unknown. Previous studies have demonstrated aberrant expression levels of circRNAs in keloid tissues $(6,7)$. Furthermore, differentially expressed circRNAs are associated with miRNA-circRNA networks that are essential during the scar wound healing process (6). Although circRNA involvement has been demonstrated during the formation of keloids (6), the detailed process and potential downstream pathways have not yet been elucidated. Among these circRNAs, circular RNA_101238 (circ_101238) is a novel molecule that is involved in the pathogenesis of several disorders, including abdominal aortic aneurysm and thoracic aortic dissection $(7,8)$. 
Circ_101238 exerts its regulatory functions via the microRNA (miRNA/miR)-320a network, and miR-320a is involved in the pathogenesis of different types of skin disorders $(9,10)$. However, the effects of circ_101238 on the pathogenesis of keloids have not yet been fully investigated. MiR-138-5p is involved in numerous biological processes, including the regulation of cancer cell proliferation and invasion (11). CDK6 is a catalytic subunit of the protein kinase complex, which is essential for $\mathrm{G}_{1}$ phase progression and $\mathrm{G}_{1} / \mathrm{S}$ transition during the cell cycle (12). By driving mTORC1 and E2F simultaneously, CyclinD-CDK6 is able to regulate cell growth and cell-cycle progression; furthermore, CDK6 inhibition reduces cell proliferation partly via TSC2 and mTORC1 (12).

The present study aimed to investigate the functional roles of circ_101238 during the growth of keloids and to determine the potential molecular mechanisms. The results demonstrated that expression levels of circ_101238 were increased in keloid samples, while circ_101238 knockdown suppressed cell proliferation and enhanced apoptosis of keloid fibroblasts by regulating miR-138-5p/CDK6 signaling. Taken together, the results of the present study suggest that the circ_101238/miR-138-5p/CDK6 axis may play a crucial role in regulation of the growth of keloid fibroblasts and that circ_101238 may act as a novel therapeutic candidate for the treatment of patients with keloids.

\section{Materials and methods}

Patient specimens. A total of 30 keloid samples were obtained from patients (19 men and 11 women; age, 22-46; mean age: $32.7 \pm 10.1$ years) who underwent surgery at the First Affiliated Hospital of Jinzhou Medical University (Jinzhou, China), between December 2017 and July 2018. Patients who received radiotherapy/chemotherapy or laser treatment prior to surgery were excluded. Patients with malignancy and immune disorder of kidney dysfunction were also excluded from the present study. Biopsies of keloids and normal $(\geq 10 \mathrm{~cm}$ from keloid) tissue samples were collected during surgery. All skin biopsies were snap-frozen in liquid nitrogen and subsequently stored at $-80^{\circ} \mathrm{C}$ until further experimentation. The present study was approved by the Ethics Committee of the First Affiliated Hospital of Jinzhou Medical University (Jinzhou, China; approval no. JYD160920). Written informed consent was provided by all patients prior to the start of the study.

Cell culture and transfection. Human keloid fibroblasts and normal fibroblasts were purchased from the Bank of Type Culture Collection of the Chinese Academy of Sciences. Samples were maintained in DMEM (Thermo Fisher Scientific, Inc.) supplemented with $10 \% \mathrm{FBS}, 100 \mathrm{U} / \mathrm{ml}$ penicillin and $100 \mu \mathrm{g} / \mathrm{ml}$ streptomycin (HyClone; GE Healthcare Life Science), at $37^{\circ} \mathrm{C}$ in a humidified atmosphere with $5 \% \mathrm{CO}_{2}$.

In order to establish the circ_101238 and CDK6 knockdown model, short hairpin (sh)RNA sequences targeting circ_101238 (sh-circ_101238; cat. no. C02097) and a control (sh-NC; cat. no. C02098) and small interfering (si)RNA against CDK6 (si-CDK6; cat. no. A01015-297) and the control (si-NC; cat. no. A01015-298) were synthesized by Shanghai GenePharma Co. Ltd. miR-138-5p mimics and inhibitors, together with the negative control (miR-NC) were also purchased from Shanghai
GenePharma Co.Ltd. In order to establish the circ_101238 and CDK6 overexpression model, wild-type (WT; LV-circ_101238 and LV-CDK6; cat. no. C02015-383 and C02015-385) or mutant (MUT; LV-NC; cat. no. C02015-384) fragments were inserted into the PLCDH-cir vector (Guangzhou Ribobio, Co., Ltd.). The quantity of each shRNA, siRNA or miRNA mimics/inhibitors was $50 \mathrm{pg} / \mathrm{ul}$ for each transfection. The lentiviral vector was generated by Hanbio Biotechnology Co., Ltd. A total of $40 \mathrm{nM}$ vector were transfected into the fibroblasts using Lipofectamine ${ }^{\circledR} 2000$ (Takara Biotechnology Co., Ltd.), according to the manufacturer's protocol. Fibroblasts were then selected using $0.5 \mu \mathrm{g} / \mathrm{ml}$ puromycin (Sigma-Aldrich; Merck $\mathrm{KGaA}$ ) for 2 weeks. The culture medium was replaced with fresh DMEM supplemented with FBS, $8 \mathrm{~h}$ post-transfection. Transfection efficiency was confirmed via reverse transcription-quantitative (RT-q)PCR analysis. The subsequent experiments were carried out $24 \mathrm{~h}$ after transfection.

$R T-q P C R$. miRNA was extracted using the miRNeasy Mini kit (Qiagen China Co., Ltd.). TaqMan microRNA assay (Applied Biosystems; Thermo Fisher Scientific, Inc.) was performed to assess miR-138-5p expression, and qPCR was subsequently performed using Applied Biosystems ${ }^{\circledR} 7500$ (Thermo Fisher Scientific Inc.).

Total RNA was extracted from tissues/cells using TRIzol ${ }^{\circledR}$ reagent (Invitrogen; Thermo Fisher Scientific, Inc.), according to the manufacturer's protocol, and protein concentration was determined using a NanoDrop 1000 spectrophotometer (Thermo Fisher Scientific, Inc.). The quality of extracted RNA was assessed using the Agilent 2100 Bioanalyzer (Agilent Technologies $\mathrm{GmbH}$ ), and the 28S:18S ratios were >1.0. cDNA was synthesized using the PrimeScript ${ }^{\mathrm{TM}}$ RT kit (Takara Biotechnology Co., Ltd.), the sample was incubated at room temperature for $30 \mathrm{~min}, 42^{\circ} \mathrm{C}$ for $45 \mathrm{mins}, 99^{\circ} \mathrm{C}$ for $5 \mathrm{mins}$ and $5^{\circ} \mathrm{C}$ for 5 mins in a PCR cycler. qPCR was subsequently performed using the SYBR Green PCR Master Mix (Takara Biotechnology Co., Ltd.), according to the manufacturer's protocols. The following primer sequences were used for qPCR: circ_101238 forward, 5'-GCTGTGAGCCGATAGCTA AC-3' and reverse, 5'-ACTCCAACACCATAGCGGAG-3'; miR-138-5p forward, 5'-AGCTGGTGTTGTGAATCAGGC CG-3' and reverse, 5'-CTCGACAACCCGCAGAGGCG-3'; CDK6 forward, 5'-TGCACAGTGTCACGAACAGAC-3' and reverse, 5'-TGAATGAAAAGCCTGCCTGGG-3'; GAPDH forward, 5'-AGAAGGCTGGGGCTCATTTG-3' and reverse, 5'-AGGGGCCATCCACAGTCTTC-3'; and U6 forward, 5'-CTCGCTTCGGCAGCACATA-3' and reverse, 5'-AAC GATTCACGAATTTGCGT-3'. The following thermocycling conditions were used for qPCR: Initial denaturation at $95^{\circ} \mathrm{C}$ for $5 \mathrm{~min}$, followed by 45 cycles of denaturation at $95^{\circ} \mathrm{C}$ for $15 \mathrm{sec}$, annealing at $60^{\circ} \mathrm{C}$ for $20 \mathrm{sec}$ and elongation at $72^{\circ} \mathrm{C}$ for $10 \mathrm{sec}$, final extension was at $72^{\circ} \mathrm{C}$ for $10 \mathrm{~min}$ Relative expression levels were calculated using the $2^{-\Delta \Delta C q}$ method (13) and normalized to the internal reference genes GAPDH (mRNA) and U6 (miRNA).

Cell proliferation assay. Cells were harvested $24 \mathrm{~h}$ post-transfection. A total of $2 \times 10^{4}$ cells were seeded into 96-well plates and the cell counting kit-8 (CCK-8) assay (Beyotime Institute of Biotechnology) was performed at day 1,2,3 and 4 following cell inoculation. Briefly, $10 \mu \mathrm{l}$ of CCK- 8 solution was added 
into each well at the corresponding time points. Following incubation at $37^{\circ} \mathrm{C}$ for $2 \mathrm{~h}$, cell proliferative activity was analyzed at a wavelength of $450 \mathrm{~nm}$, using a microplate reader (Bio-Rad Laboratories, Inc.).

Flow cytometric analysis of apoptosis. Transfected cells were placed into 6 -well plates at a density of $2 \times 10^{5}$ cells/well and centrifugated at $5,000 \mathrm{x} \mathrm{g}$ for $5 \mathrm{~min}$ at room temperature. Cell pellets were rinsed with PBS and re-suspended into the wells. Cells were incubated with $5 \mu$ l propodium iodide (Thermo Fisher Scientific) in the dark for $30 \mathrm{~min}$ at $4^{\circ} \mathrm{C}$ and subsequently stained with $5 \mu$ l Annexin V-FITC for $15 \mathrm{~min}$ at room temperature (cat. no. ab1408; Abcam). Apoptotic cells were subsequently analyzed using a flow cytometer (BD Biosciences) and FlowJo software (version 7.6; FlowJo LLC).

Bioinformatic analysis and dual-luciferase reporter assay. The Targetscan (www.targetscan.org) and miRanda (www. microrna.org/microrna) databases were used to predict the potential targets of circ_101238 and miR-138-5p.

The WT fragments of the 3'-untranslated region (UTR) of circ_101238/CDK6, with the predicted binding sites of miR-138-5p, were purchased from Shanghai GenePharma Co., Ltd. The fragments were cloned into the pmirGLO Dual-Luciferase miRNA Target Expression vector (Promega Corporation), according to the manufacturer's protocol. The circ_101238/CDK6-3'-UTR-MUT plasmid containing the mutant miR-138-5p binding site was generated using the QuikChange Multi Site-Directed Mutagenesis kit (Agilent Technologies, Inc.). The corresponding vectors were subsequently used to co-transfect 293 cells (Bank of Type Culture Collection of the Chinese Academy of Sciences) with miR-138-5p mimics or the control using Lipofectamine ${ }^{\circledR}$ 2000 (Takara Biotechnology Co., Ltd.). Following incubation for $48 \mathrm{~h}$ at $37^{\circ} \mathrm{C}$ with $5 \% \mathrm{CO}_{2}$, firefly and Renilla luciferase activities were detected using the Dual-Luciferase Reporter Assay System (Promega Corporation), according to the manufacturer's protocol. Firefly luciferase activity was normalized to Renilla luciferase activity.

Western blotting. Total protein was extracted from fibroblasts using radioimmunoprecipitation assay buffer (Beyotime Institute of Biotechnology). Protein concentration was measured using a bicinchoninic assay (Beyotime Institute of Biotechnology) and $30 \mu \mathrm{g}$ protein/lane was separated via SDS-PAGE using 10\% gels. The separated proteins were subsequently transferred onto nitrocellulose membranes (EMD Millipore) and blocked with TBS containing 5\% skimmed milk at room temperature for $1 \mathrm{~h}$. The membranes were incubated with primary antibodies against: CDK6 (dilution 1:3,000; cat. no. ab151247; Abcam), caspase-3 (dilution 1:500; cat. no. ab4051; Abcam) and GAPDH (dilution 1:10,000; cat. no. ab8245; Abcam) overnight at $4^{\circ} \mathrm{C}$. Following the primary incubation, membranes were incubated with horseradish peroxidase-conjugated anti-rabbit (dilution 1:5,000; cat. no. sc-2357; Santa Cruz Biotechnology, Inc.) or anti-mouse IgG (dilution 1:5,000; cat. no. sc-2371; Santa Cruz Biotechnology, Inc.) secondary antibodies at room temperature for $1 \mathrm{~h}$. Protein bands were visualized using the enhanced chemiluminescence protein detection kit (Pierce; Thermo
Fisher Scientific, Inc.), and the signal was analyzed using Image J software (version 1.48; National Institutes of Health).

Statistical analysis. Statistical analysis was performed using SPSS software 21.0 (IBM Corp.) and data are presented as the mean \pm standard deviation. Paired Student's t-test was used to compare differences between two groups, while ANOVA, followed by Dunnett's post-hoc test were used to compare differences between multiple groups against the control. Pearson's correlation analysis was performed to determine the correlation between RNA expression levels. All experiments were performed in triplicate. $\mathrm{P}<0.05$ was considered to indicate a statistically significant difference.

\section{Results}

Circ_101238 expression is upregulated in keloids and fibroblasts. The expression levels of circ_101238 were determined in 30 keloid and matched adjacent normal tissue samples via RT-qPCR analysis. The results demonstrated that circ_101238 expression was significantly upregulated in keloid samples compared with the control group $(\mathrm{P}<0.05$; Fig. 1A). Similarly, upregulated circ_101238 expression was also detected in keloid fibroblasts compared with the control group ( $\mathrm{P}<0.05$; Fig. 1B). In order to perform the gain- and loss-of-function experiments, circ_101238 knockdown and overexpression models were established by transfecting fibroblasts with sh-circ_101238 and LV-circ_101238, respectively. Transfection efficiencies were confirmed via RT-qPCR analysis $(\mathrm{P}<0.05$; Fig. $1 \mathrm{C}$ and $\mathrm{D})$.

Circ_101238 knockdown inhibits growth, while promoting apoptosis of keloid fibroblasts. In order to investigate the regulatory functions of circ_101238 on the proliferation and apoptosis of keloid fibroblasts, circ_101238 was knocked down in fibroblasts using lentiviral vectors. The CCK-8 assay demonstrated that the proliferative ability of keloid fibroblasts transfected with sh-circ_101238 significantly decreased compared with the control (sh-NC) group $(\mathrm{P}<0.05$; Fig. 1E). Furthermore, mRNA and protein expression levels of the proliferation-associated molecule, CDK6 were significantly decreased following transfection with sh-circ_101238 $(\mathrm{P}<0.05$; Fig. $1 \mathrm{~F}$ and $\mathrm{G})$. Apoptosis was also assessed in transfected cells via flow cytometric analysis, and the results indicated that the apoptotic rate was significantly increased in keloid fibroblasts transfected with sh-circ_101238 compared with the sh-NC group ( $\mathrm{P}<0.05$; Fig. $1 \mathrm{H}$ and $\mathrm{I})$. Furthermore, mRNA and protein expression levels of the apoptosis marker, caspase-3 were significantly increased following transfection with sh-circ_101238 ( $\mathrm{P}<0.05$; Fig. 1J-L). Taken together, these results suggest that the growth of keloid fibroblasts may be inhibited by knockdown of circ_101238 expression.

miR-138-5p is the putative target of circ_101238 in keloids. In order to confirm whether circ_101238 is a novel oncogenic factor in keloids, and determine its potential roles through targeting downstream miRNAs, the complementary binding sites between miR-138-5p and circ_101238 were predicted via bioinformatics analysis (Fig. 2A). The association between circ_101238 and miR-138-5p was verified via the dual-luciferase reporter assay, in which luciferase reporters containing WT 
A
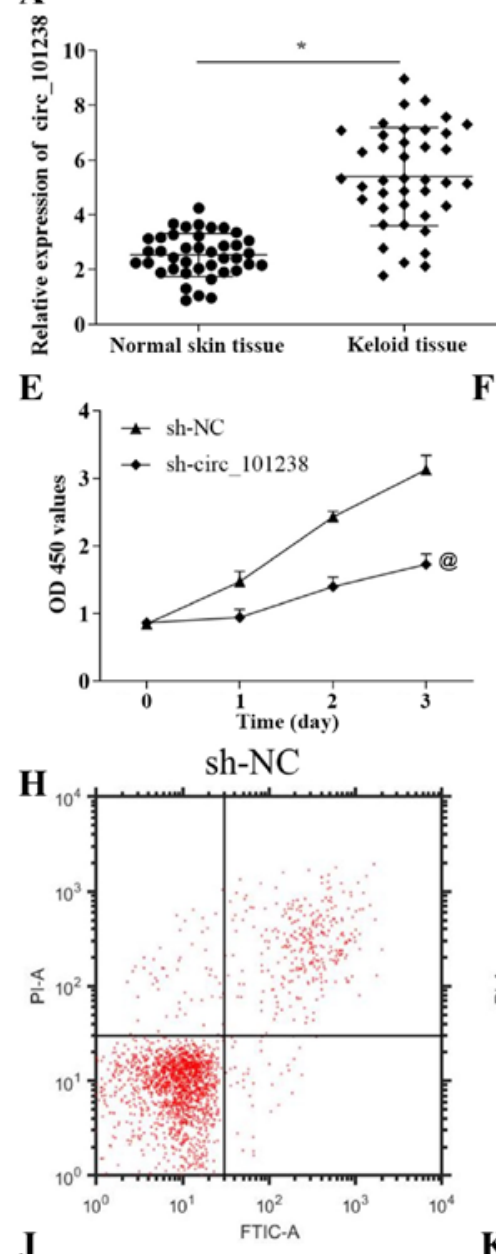

J

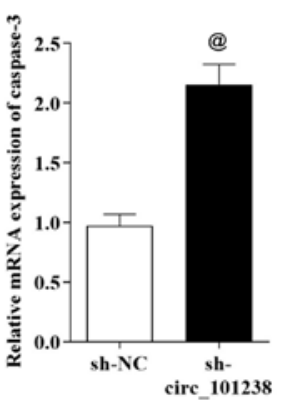

B
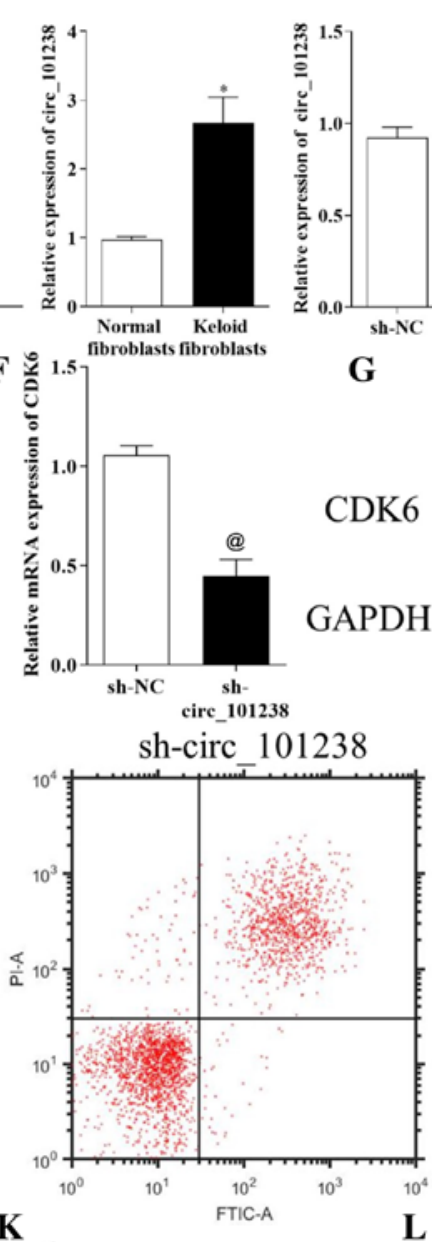

$\mathbf{K}$

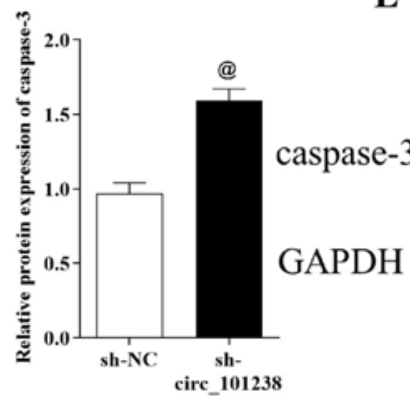

D
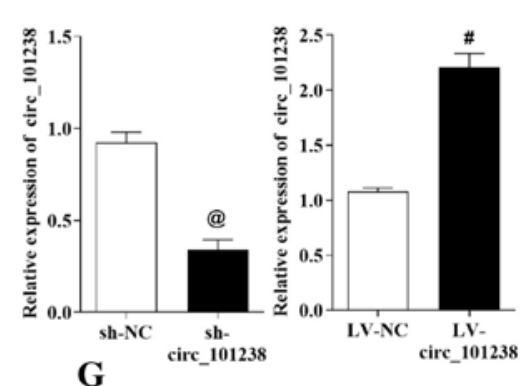

sh-NC sh-circ_101238

CDK6

GAPDH

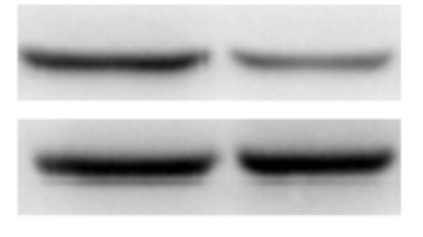

I

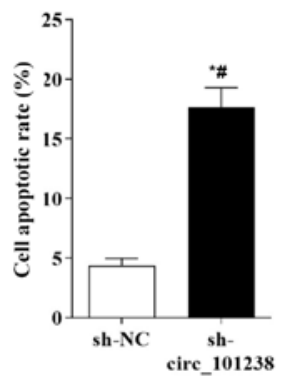

sh-NC sh-circ_101238

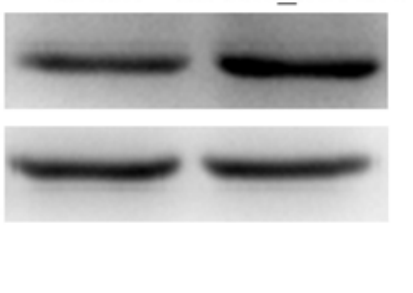

Figure 1. Circ_101238 expression is significantly upregulated in keloid samples and fibroblasts, while circ_101238 knockdown suppressed the growth of keloid fibroblasts. (A) Circ_101238 expression levels were determined in 30 keloid tissues and matched adjacent normal controls via RT-qPCR analysis. (B) Circ_101238 expression levels were assessed in normal and keloid fibroblasts. The transfection efficiencies of (C) sh-circ_101238 and (D) LV-circ_101238 were confirmed via RT-qPCR analysis. (E) The proliferative ability of keloid fibroblasts transfected with sh-circ_101238 was significantly inhibited. (F) The mRNA levels of CDK6 were downregulated in cells transfected with sh-circ_101238. (G) The protein levels of CDK6 were reduced in transfected cells. (H) Cell apoptosis was significantly enhanced in keloid cells following transfection with sh-circ_101238. (I) Cell apoptotic rate was increased in transfected cells. (J) The mRNA levels of caspase-3 were elevated following the transfection with sh-circ_101238. (K) The protein levels of caspase-3 were enhanced in cells transfected with sh-circ_101238. (L) Western blotting of caspase-3 in transfected cells. All experiments were performed in triplicate. * $\mathrm{P}<0.05$ vs. normal samples, ${ }^{\#} \mathrm{P}<0.05$ vs. LV-NC, ${ }^{\circledR} \mathrm{P}<0.05$ vs. sh-NC. Circ, circular RNA; RT-qPCR, reverse transcription-quantitative PCR; sh, short hairpin; NC, negative control; OD, optical density.

(circ_101238-WT) or MUT (circ_101238-MUT) fragments of potential miR-138-5p binding sites were generated. The results demonstrated that miR-138-5p mimics significantly suppressed luciferase activity of the circ_101238-WT vector compared with the control $(\mathrm{P}<0.05$; Fig. 2B). The expression level of miR-138-5p was significantly increased in keloid fibroblasts transfected with sh-circ_101238 and the levels of miR-138-5p were reduced in cells transfected with LV-circ_101238 $(\mathrm{P}<0.05$; Fig. 2C and D). Furthermore, expression levels of miR-138-5p were significantly decreased in keloid samples compared with the control group, where the levels of circ_101238 and miR-138-5p were inversely correlated $(\mathrm{P}<0.05$; Fig. $2 \mathrm{E}$ and $\mathrm{F})$.

miR-138-5p is involved in circ_101238-modulated cell growth of keloid fibroblasts. Cells were transfected with miR-138-5p mimics or inhibitors and the transfection efficiencies were confirmed via RT-qPCR analysis ( $\mathrm{P}<0.05$; Fig. $2 \mathrm{G}$ ). Transfection with miR-138-5p inhibitors significantly induced 
A

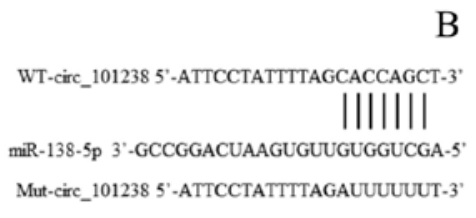

$\mathbf{E}$

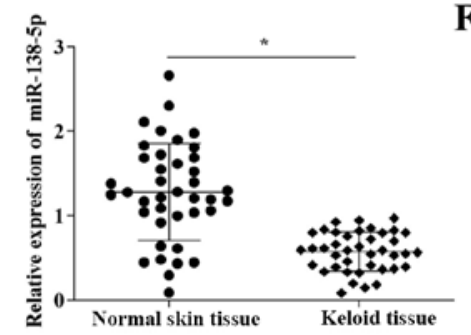

H

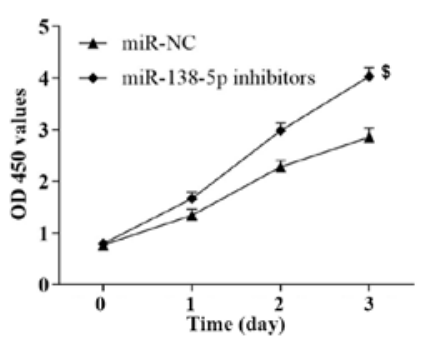

$\mathbf{K}$

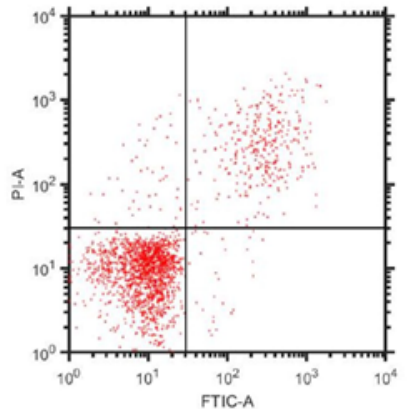

M

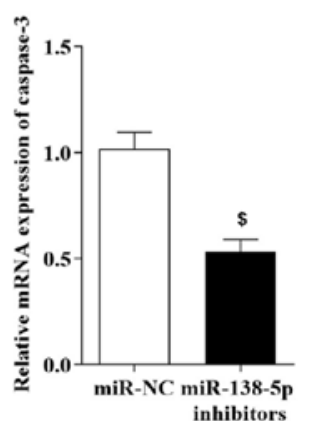

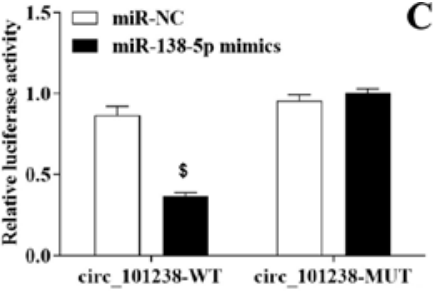

$\mathbf{F}$

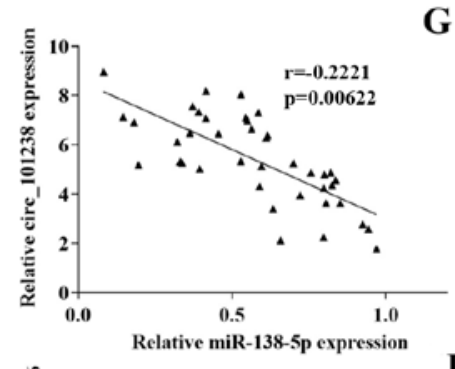

I

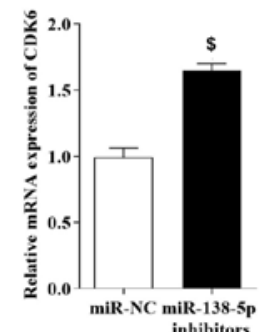

miR-138-5p inhibitors

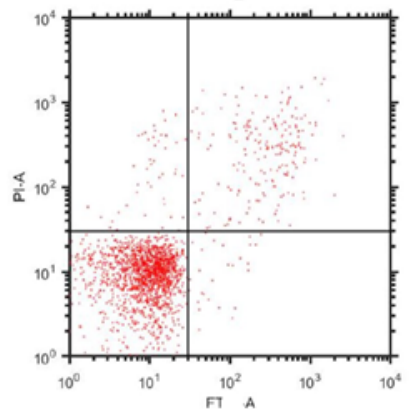

$\mathbf{N}$

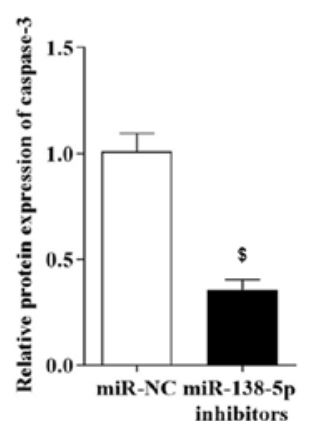

C

$\mathbf{J}$

$\mathbf{L}$

$\mathbf{O}$

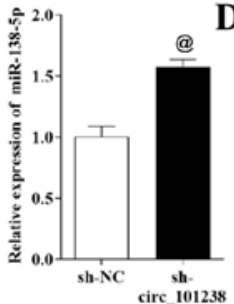

G
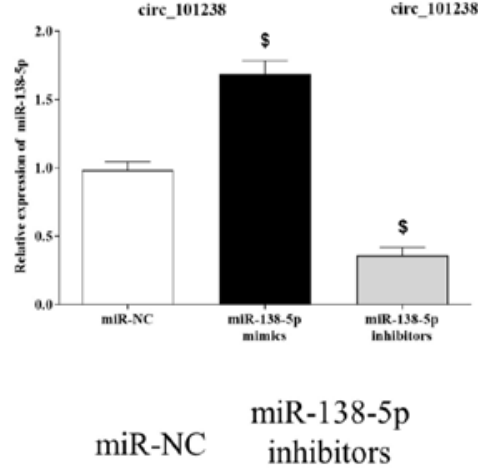

CDK6

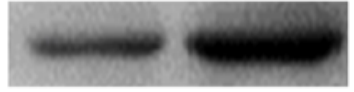

GAPDH
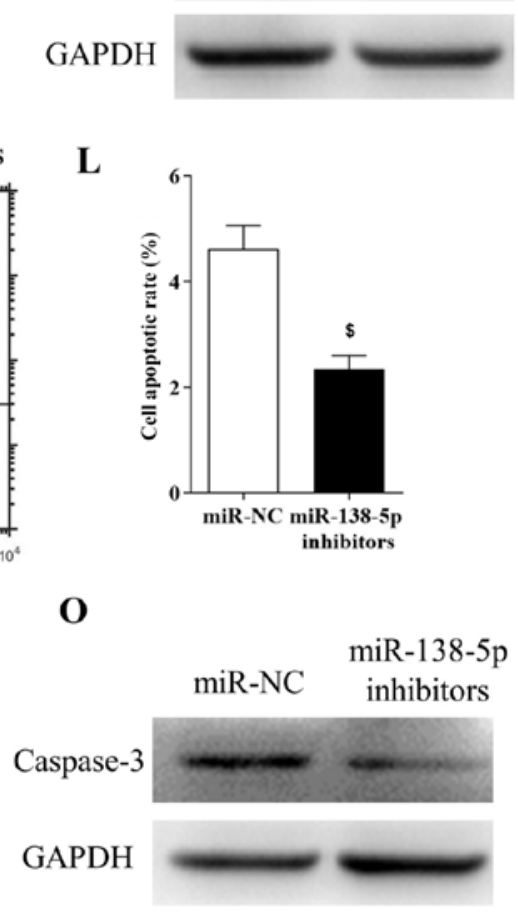

Figure 2. miR-138-5p is a putative target of circ_101238 in keloid tissue and is involved in circ_101238-regulated cell growth of keloid fibroblasts. (A) The complementary binding sites between miR-138-5p and circ_101238 were predicted via bioinformatic analysis. (B) Treatment with miR-138-5p notably decreased luciferase activity of circ_101238-WT compared with the mutant. (C) miR-138-5p expression levels significantly increased in keloid cells transfected with sh-circ_101238 compared to sh-NC. (D) The levels of miR-138-5p were reduced following the transfection with LV-circ_101238. (E) miR-138-5p expression significantly decreased in keloid samples compared to normal skin. (F) The levels of circ_101238 and miR-138-5p were inversely correlated in keloid tissue $(\mathrm{r}=-0.2221 ; \mathrm{P}=0.00622)$. (G) The transfection efficiencies of miR-138-5p mimics and inhibitors were confirmed via reverse transcription-quantitative PCR analysis. (H-J) The proliferative ability of keloid fibroblasts significantly enhanced following transfection with miR-138-5p inhibitors. (K-O) Cell apoptosis significantly decreased following transfection with miR-138-5p inhibitors compared to miR-NC. All experiments were performed in triplicate. " $\mathrm{P}<0.05 \mathrm{vs}$. normal samples, ${ }^{\prime \prime} \mathrm{P}<0.05$ vs. LV-NC, ${ }^{\circledR} \mathrm{P}<0.05$ vs. sh-NC, ${ }^{\$} \mathrm{P}<0.05$ vs. miR-NC. miR, microRNA; circ, circular RNA; sh, short hairpin; Mut, mutant; WT, wild type; NC, negative control; OD, optical density; CDK6, cyclin-dependent kinase 6.

proliferation compared with the control group $(\mathrm{P}<0.05$; Fig. $2 \mathrm{H})$. Furthermore, mRNA and protein expression levels of CDK6 were significantly increased in keloid fibroblasts transfected with miR-138-5p inhibitors compared with the control group $(\mathrm{P}<0.05$; Fig. 2I and J). Apoptosis was also assessed in the transfected cells, and the results demonstrated that the apoptotic 
A

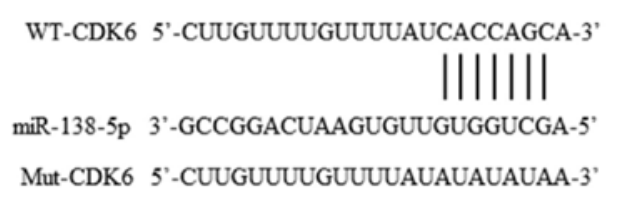

C

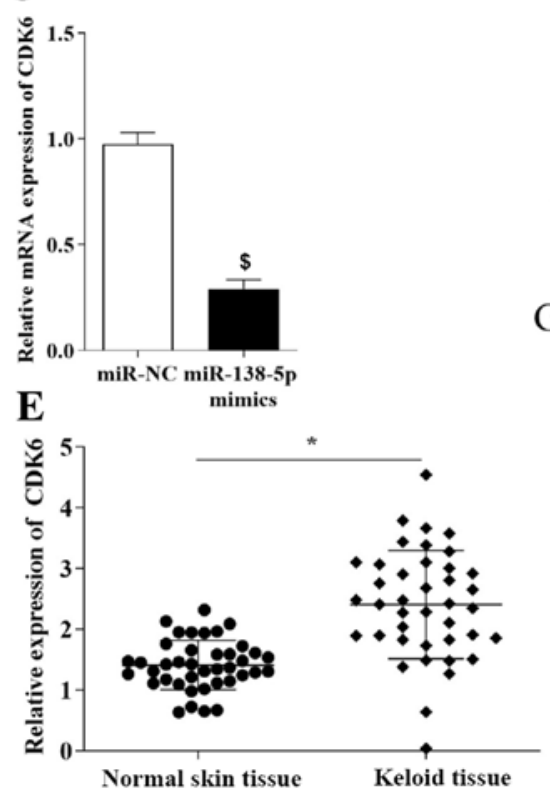

B

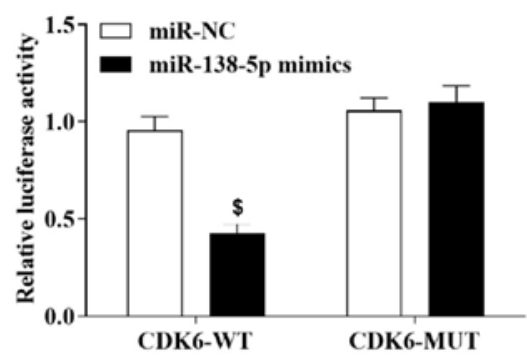

D

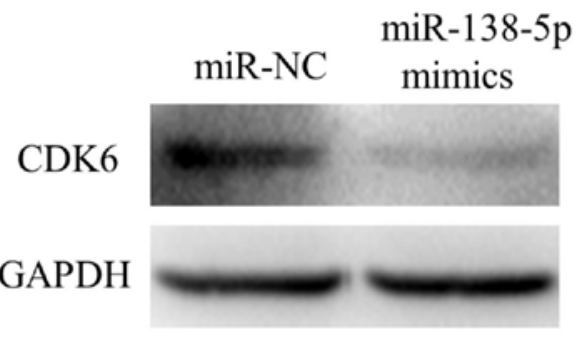

$\mathbf{F}$

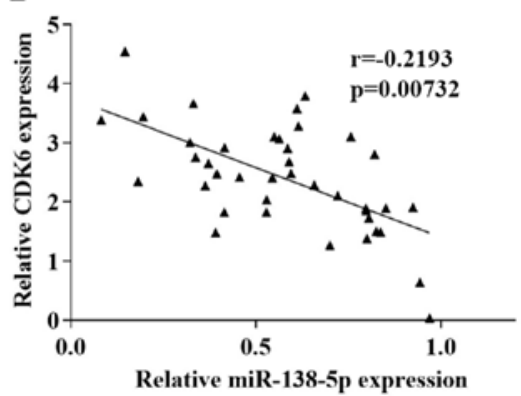

Figure 3. CDK6 is a promising downstream molecule of miR-138-5p. (A) The binding sites of miR-138-5p and CDK6 transcripts were predicted via bioinformatic analysis. (B) The association between miR-138-5p and CDK6 was confirmed via the dual-luciferase activity assay. (C) The mRNA levels of CDK6 were downregulated in keloid fibroblasts following transfection with miR-138-5p mimics. (D) The protein levels of CDK6 were reduced in cells transfected with miR-138-5p mimics. (E) In comparison with normal skin, CDK6 expression was significantly upregulated in keloid tissues. (F) The levels of miR-138-5p and CDK6 were inversely correlated in keloid samples $(\mathrm{r}=-0.2193$; $\mathrm{P}=0.00732)$. All experiments were performed in triplicate. ${ }^{*} \mathrm{P}<0.05$ vs. normal samples, ${ }^{\$} \mathrm{P}<0.05$ vs. miR-NC. CDK6, cyclin-dependent kinase 6; miR, microRNA; WT, wild type; Mut, mutant; NC, negative control.

rate was significantly decreased following transfection with miR-138-5p inhibitors $(\mathrm{P}<0.05$; Fig. $2 \mathrm{~K}$ and $\mathrm{L})$, which was confirmed by downregulated expression levels of caspase-3 (P<0.05; Fig. 2M-O).

CDK6 is a novel downstream molecule of miR-138-5p. In order to identify the promising targets of miR-138-5p, the binding sites on miR-138-5p and CDK6 transcripts were predicted (Fig. 3A). The association between miR-138-5p and CDK6 was verified via the dual-luciferase reporter assay, in which luciferase vectors containing WT (CDK6-WT) or MUT (CDK6-MUT) fragments of predicted miR-138-5p binding sites were generated. The results demonstrated that miR-138-5p mimics significantly suppressed luciferase activity of the plasmids containing CDK6-WT, but not those containing CDK6-MUT (P<0.05; Fig. 3B). Furthermore, RT-qPCR and western blot analyses indicated that CDK6 expression levels were downregulated in keloid fibroblasts transfected with miR-138-5p mimics compared with the control group $(\mathrm{P}<0.05$; Fig. 3C and D). Notably, the expression levels of miR-138-5p were significantly increased in the keloid tissues compared with the control group, where the levels of miR-138-5p and CDK6 were inversely correlated $(\mathrm{P}<0.05$; Fig. $3 \mathrm{E}$ and $\mathrm{F})$.
Circ_101238 affects the proliferation and apoptosis of keloid fibroblasts via the miR-138-5p/CDK6 axis. In order to further identify the effects of miR-138-5p and CDK6 in circ_101238-regulated biological behavior changes within keloidfibroblasts,cellswereco-transfectedwithLV-circ_101238 and miR-138-5p mimics or miR-138-5p mimics/inhibitors and si-CDK6/LV-CDK6, respectively. The results indicated that the enhanced cell proliferation and inhibition of apoptosis induced by LV-circ_101238 were significantly counteracted following transfection with miR-138-5p mimics $(\mathrm{P}<0.05$; Fig. 4A-C). Furthermore, enhanced cell proliferation induced by miR-138-5p inhibitors was significantly reversed following CDK6 knockdown, whereas the effects on cell proliferation caused by miR-138-5p mimics were significantly attenuated following overexpression of CDK6 ( $\mathrm{P}<0.05$; Fig. 4D-I). Taken together, these results suggest that circ_101238 regulates the growth of keloid fibroblasts via miR-138-5p/CDK6 signaling.

\section{Discussion}

A Previous study focused on the regulatory roles of non-coding RNAs during the initiation and progression of keloids (10) and microarray data have indicated aberrantly expressed long 
A

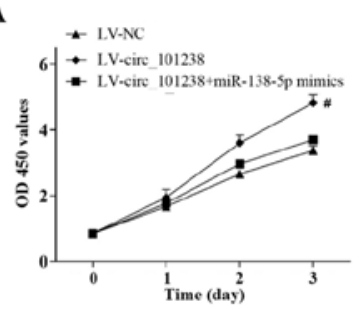

D

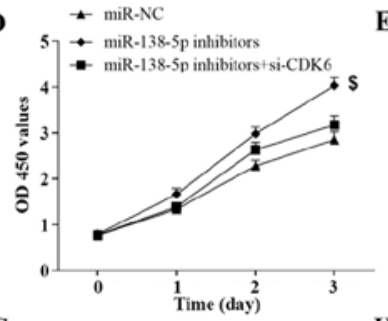

G

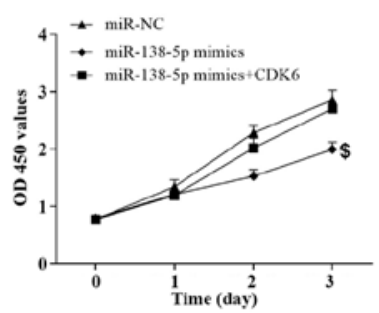

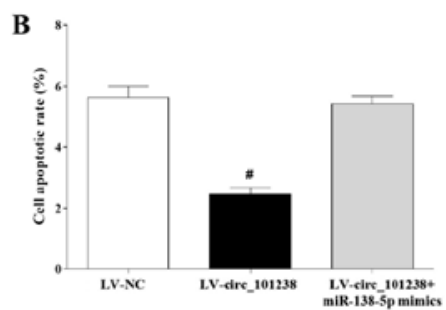

C

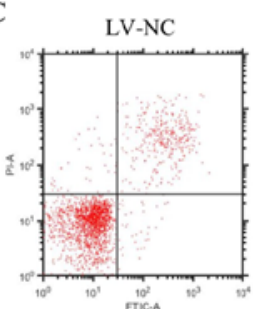

E :

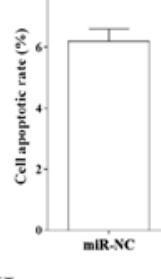

H

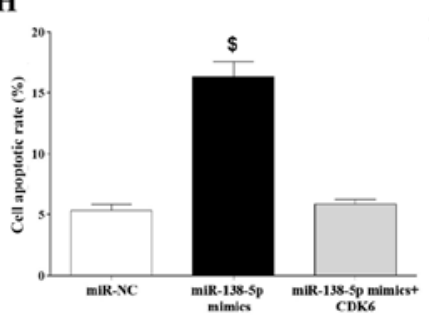

F
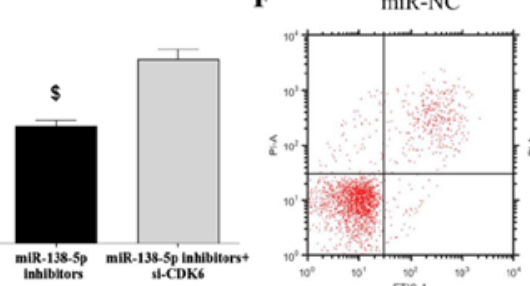

I

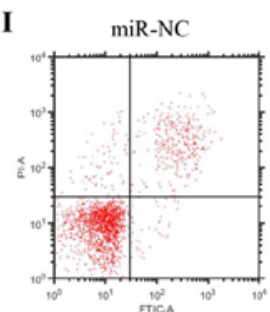

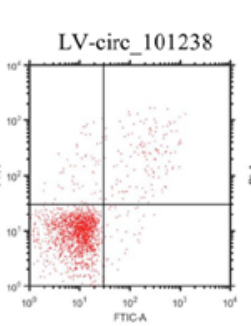

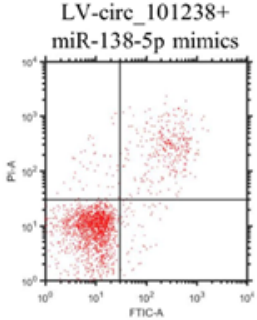

miR-138-5p inhibitors

miR-138-5p inhibitors + si-CDK6
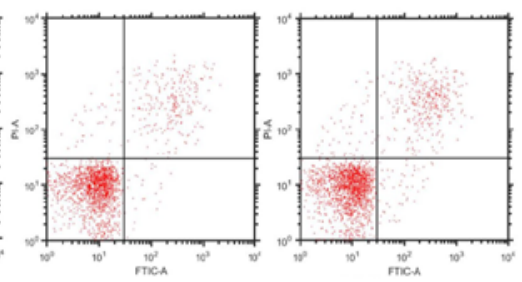

miR-138-5p mimics

miR-138-5p mimics
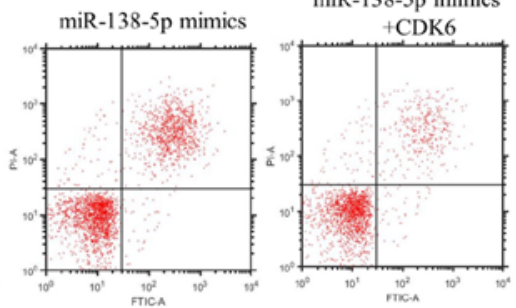

Figure 4. Circ_101238 influences the growth of keloid fibroblasts via the miR-138-5p/CDK6 signaling pathway. (A-C) The effects on cell proliferation and apoptosis induced by LV-circ_101238 were reversed following transfection with miR-138-5p mimics. (D-F) Enhanced cell proliferation caused by transfection with miR-138-5p inhibitors was reversed following CDK6 knockdown. (G-I) The effects induced by transfection with miR-138-5p mimics were counteracted following overexpression of CDK6. All experiments were performed in triplicate. ${ }^{\text {} P}<0.05$ vs. LV-NC, ${ }^{\$} \mathrm{P}<0.05$ vs. miR-NC. Circ, circular RNA; miR, microRNA; CDK6, cyclin-dependent kinase 6; NC, negative control; OD, optical density.

non-coding (lnc)RNAs and miRNAs in keloid tissues (14). Furthermore, a previous study reported that circRNAs are aberrantly expressed in keloid fibroblasts compared with healthy controls (15). A complex network of circRNAs and miRNAs are involved in the keloid scarring process (12), suggesting involvement of differentially expressed circRNAs in the scar wound healing process (6). Previous studies have demonstrated that circRNAs function as miRNA 'sponges' that competitively inhibit the activity of corresponding miRNAs $(4,16)$. miRNAs are small non-coding RNAs with a length of $\sim 22$ nucleotides, which are novel downstream targets of other non-coding RNAs, such as lncRNAs and circRNAs. It has been suggested that miRNAs function by binding to the 3'-UTR of their target mRNAs and subsequently affect several biological behaviors, including cell proliferation, migration, invasion and apoptosis (4). Notably, a previous study indicated that miR-196a is involved in the formation of keloids (17). However, the detailed functions of circRNAs and miRNAs during the initiation and development of keloids are not yet fully understood. Thus, the present study aimed to investigate the effects of circRNA and miRNA on the proliferation and apoptosis of keloid fibroblasts.

The results of the present study indicated that the expression levels of circ_101238 and CDK6 remarkedly increased in keloid tissues, while the expression levels of miR-138-5p notably decreased. Furthermore, circ_101238 was demonstrated to bind to miR-138-5p, which subsequently targets CDK6. Following transfection with sh-circ_101238 or miR-138-5p mimics, the proliferative abilities and expression levels of
CDK6 significantly decreased in keloid fibroblast, while cell apoptosis was notably enhanced. These results suggest that circ_101238 may affect the growth of keloids by regulating miR-138-5p/CDK6 signaling. Similarly, previous studies have reported that non-coding RNAs, including miR-152-3p, miR-21 and miR-203 are differently expressed in keloid samples, which may contribute to the formation of keloids (18-20). Furthermore, IncRNA HOXA11-AS has been demonstrated to regulate the progression of keloids via miR-124-3p/Smad5 signaling (21). A recent study reported the involvement of the circRNAs and miRNAs regulatory networks during keloid scarring (22).

The present study also aimed to identify the molecular mechanisms underlying circ_101238-modulated cell proliferation and apoptosis in keloid fibroblasts. Co-transfection with miR-138-5p mimics reversed the effects induced by overexpressing circ_101238. Furthermore, transfection with si-CDK6 attenuated the effects induced by miR-138-5p inhibitors in keloid fibroblasts, while transfection with LV-CDK6 counteracted the effects induced by miR-138-5p mimics. Taken together, the results of the present study confirmed that miR-138-5p and CDK6 are involved in circ_101238-regulated biological behavior changes within keloid fibroblasts. CDK6 is regulated by cyclin D and associated with cell proliferation via regulation of cell cycle progression (12). Consistent with the results of the present study, previous findings have demonstrated the essential roles of CDK6, which is involved in several signaling pathways associated with the growth of keloid fibroblasts and scarring (23-26). 
In conclusion, the results of the present study demonstrated that circ_101238 expression increased in keloid tissues, while knockdown of circ_101238 suppressed cell proliferation and promoted the apoptosis of keloid fibroblasts by regulating the miR-138-5p/CDK6 axis. However, there were limitations to the present study, for example, in vivo work should be performed to confirm existing findings in future study, for example, to elucidate the effects of circ_101238 knockdown on the expression of downstream molecules such as CDK6 and caspase-3; furthermore, other assays such as immunocytostaining could be carried out to evaluate the expression of associated proteins including CDK6 and caspase-3. These results suggest that the novel circ_101238/miR-138-5p/CDK6 signaling pathway may play vital roles during the growth of keloids, and circ_101238 may act as a promising candidate for the targeted therapy of patients with keloids.

\section{Acknowledgements}

Not applicable.

\section{Funding}

No funding was received.

\section{Availability of data and materials}

The datasets used and/or analysed during the current study are available from the corresponding author on reasonable request.

\section{Authors' contributions}

ND designed the present study. DY, ML and ND performed the experiments and analyzed the data. All authors read and approved the final manuscript.

\section{Ethics approval and consent to participate}

The present study was approved by the Ethics Committee of the First Affiliated Hospital of Jinzhou Medical University (Jinzhou, China; approval no. JYD160920). Written informed consent was provided by all patients prior to the study start.

\section{Patient consent for publication}

Not applicable.

\section{Competing interests}

The authors declare that they have no competing interests.

\section{References}

1. Jfri A, O'Brien E, Alavi A and Goldberg SR: Association of hidradenitis suppurativa and keloid formation: A therapeutic challenge. JAAD Case Rep 5: 675-678, 2019.

2. Betarbet $U$ and Blalock T: Keloids: A review of etiology, prevention, and treatment. J Clin Aesthet Dermatol 13: 33-43, 2020

3. Lim KH, Itinteang T, Davis PF and Tan ST: Stem cells in keloid lesions: A review. Plast Reconstr Surg Glob Open 7: e2228, 2019.
4. Memczak S, Jens M, Elefsinioti A, Torti F, Krueger J, Rybak A, Maier L, Mackowiak SD, Gregersen LH, Munschauer M, et al: Circular RNAs are a large class of animal RNAs with regulatory potency. Nature 495: 333-338, 2013.

5. Vicens Q and Westhof E: Biogenesis of circular RNAs. Cell 159: 13-14, 2014

6. Wang J, Wu H, Xiao Z and Dong X: Expression profiles of lncRNAs and circRNAs in keloid. Plast Reconstr Surg Glob Open 7: e2265, 2019.

7. Kumar S, Boon RA, Maegdefessel L, Dimmeler S and Jo H: Role of noncoding RNAs in the pathogenesis of abdominal aortic aneurysm. Circ Res 124: 619-630, 2019.

8. Zou M, Huang C, Li X, He X, Chen Y, Liao W, Liao Y, Sun J, Liu Z, Zhong L and Bin J: Circular RNA expression profile and potential function of hsa_circRNA_101238 in human thoracic aortic dissection. Oncotarget 8: 81825-81837, 2017.

9. Singhvi G, Manchanda P, Krishna Rapalli V, Kumar Dubey S, Gupta G and Dua K: MicroRNAs as biological regulators in skin disorders. Biomed Pharmacother 108: 996-1004, 2018.

10. Zhang GY, Wu LC, Liao T, Chen GC, Chen YH, Zhao YX, Chen SY, Wang AY, Lin K, Lin DM, et al: A novel regulatory function for miR-29a in keloid fibrogenesis. Clin Exp Dermatol 41: 341-345, 2016.

11. Zhu D, Gu L, Li Z, Jin W, Lu Q and Ren T: MiR-138-5p suppresses lung adenocarcinoma cell epithelial-mesenchymal transition, proliferation and metastasis by targeting ZEB2. Pathol Res Pract 215: 861-872, 2019.

12. Romero-Pozuelo J, Figlia G, Kaya O, Martin-Villalba A and Teleman AA: CDK4 and CDK6 couple the cell-cycle machinery to cell growth via mTORC1. Cell Rep 31: 107504, 2020.

13. Livak KJ and Schmittgen TD: Analysis of relative gene expression data using real-time quantitative PCR and the 2(-Delta Delta C(T)) method. Methods 25: 402-408, 2001.

14. Liang $X$, Ma L, Long $X$ and Wang X: LncRNA expression profiles and validation in keloid and normal skin tissue. Int $\mathrm{J}$ Oncol 47: 1829-1838, 2015.

15. Zhang Z, Yu K, Liu O, Xiong Y, Yang X, Wang S, Zhang S, Feng $Y$ and Peng Y: Expression profile and bioinformatics analyses of circular RNAs in keloid and normal dermal fibroblasts. Exp Cell Res 388: 111799, 2020.

16. Hansen TB, Jensen TI, Clausen BH, Bramsen JB, Finsen B, Damgaard CK and Kjems J: Natural RNA circles function as efficient microRNA sponges. Nature 495: 384-388, 2013.

17. Kashiyama K, Mitsutake N, Matsuse M, Ogi T, Saenko VA, Ujifuku K, Utani A, Hirano A and Yamashita S: miR-196a downregulation increases the expression of type I and III collagens in keloid fibroblasts. J Invest Dermatol 132: 1597-1604, 2012.

18. Wang R, Bai Z, Wen X, Du H, Zhou L, Tang Z, Yang Z and Ma W: MiR-152-3p regulates cell proliferation, invasion and extracellular matrix expression through by targeting FOXF1 in keloid fibroblasts. Life Sci 234: 116779, 2019.

19. Shi K, Qiu X, Zheng W, Yan D and Peng W: MiR-203 regulates keloid fibroblast proliferation, invasion, and extracellular matrix expression by targeting EGR1 and FGF2. Biomed Pharmacother 108: 1282-1288, 2018.

20. Wu J, Fang L, Cen Y, Qing Y, Chen J and Li Z: MiR-21 regulates keloid formation by downregulating Smad7 via the TGF- $\beta / S m a d$ signaling pathway. J Burn Care Res 40: 809-817, 2019.

21. Jin J, Zhai HF, Jia ZH and Luo XH: Long non-coding RNA HOXA11-AS induces type I collagen synthesis to stimulate keloid formation via sponging miR-124-3p and activation of Smad5 signaling. Am J Physiol Cell Physiol 317: C1001-C1010, 2019.

22. Shi J, Yao S, Chen P, Yang Y, Qian M, Han Y, Wang N, Zhao Y, He Y, Lyu $\mathrm{L}$ and $\mathrm{Lu} \mathrm{D}$ : The integrative regulatory network of circRNA and microRNA in keloid scarring. Mol Biol Rep 47: 201-209, 2020.

23. Cai Y,Zhu S, Yang W, Pan M, Wang C and Wu W: Downregulation of $\beta$-catenin blocks fibrosis via Wnt 2 signaling in human keloid fibroblasts. Tumour Biol 39: 1010428317707423, 2017.

24. Harmanci D, Erkan EP, Kocak A and Akdogan GG: Role of the microRNA-29 family in fibrotic skin diseases. Biomed Rep 6: 599-604, 2017.

25. Limandjaja GC, Belien JM, Scheper RJ, Niessen FB and Gibbs S: Hypertrophic and keloid scars fail to progress from the CD34-/ $\alpha-$ smooth muscle actin $(\alpha-S M A)+$ immature scar phenotype and show gradient differences in $\alpha$-SMA and p16 expression. Br J Dermatol 182: 974-986, 2020.

26. Sherr CJ, Beach D and Shapiro GI: Targeting CDK4 and CDK6: From discovery to therapy. Cancer Discov 6: 353-367, 2016. 